DOI: 10.17234/SRAZ.65.56

\title{
Lettere di Laura Zanon Paladini a Giuseppe Sabalich nella Biblioteca scientifica di Zara
}

\author{
Ana Bukvić \\ Andrijana Jusup Magazin \\ Università di Zara \\ abukvic@unizd.hr \\ ajusup@unizd.hr
}

\begin{abstract}
Nella collezione di manoscritti della Biblioteca scientifica di Zara si conserva il carteggio di Giuseppe Sabalich. Nell'epistolario, che si rivela una ricca miscellanea di lettere private e ufficiali, sono conservate cinque lettere e 26 cartoline postali dell'attrice italiana Laura Zanon Paladini, scritte tra il 29 giugno del 1898 e il 14 agosto del 1906 e indirizzate ad uno tra i più eminenti intellettuali zaratini che scrive in italiano a cavallo tra l'Ottocento e il Novecento. L'epistolario non costituisce solo una documentazione relativa "al privato" dell'autrice, ma costituisce una fonte primaria per individuare la tipologia relazionale tra Giuseppe Sabalich e Laura Zanon Paladini, ispiratrice dei suoi monologhi e interprete del suo teatro. Obiettivo di questa ricerca è analizzare i documenti: le caratteristiche generali delle lettere, i contenuti delle lettere e la contestualizzazione delle stesse sia nell'epistolario che nell'ambito dell'attività teatrale di Giuseppe Sabalich.
\end{abstract}

Parole chiave: Giuseppe Sabalich, Laura Zanon Paladini, epistolario, teatro dialettale

La ricerca sulle lettere di Laura Zanon Paladini a Giuseppe Sabalich fa parte del progetto di valorizzazione del carteggio inedito di Giuseppe Sabalich, conservato nel Fondo Giuseppe Sabalich nella collezione di manoscritti della Biblioteca Scientifica di Zara. La ragione per cui è stata condotta questa ricerca è la necessità di far luce sulla bio-bibliografia dell'intellettuale, di completare gli studi e le ricerche precedenti e di documentare i legami storici, culturali e letterari tra le due sponde dell' Adriatico. Il carteggio si presenta come una vera e propria miscellanea di lettere private e ufficiali riguardanti un arco temporale che va dagli anni Ottanta dell'Ottocento fino agli anni Venti del Novecento. Si tratta di un documento di alto valore scientifico, storico e culturale che si presta a diversi livelli di lettura; offre, infatti, nuovi spunti di ricerca agli studiosi e aggiunge nuovi tasselli utili per lo studio dell'ambiente letterario-culturale a Zara 
negli anni precedenti alla Prima guerra mondiale, il cui maggiore protagonista era, appunto, Giuseppe Sabalich, secondo Narciso Detoni: «un artista di Zara, un artista per Zara» (Detoni, 1980: 265). Rinomato storico, narratore, poeta e scrittore di teatro, Giuseppe Sabalich (Zara, 1856 - Zara, 1928) è l'autore più prolifico in lingua italiana a Zara a cavallo tra l'Ottocento e il Novecento. Scrisse studi storici e folcloristici, raccolte di poesia e più di settanta commedie, monologhi e drammi (Teatro, I monologhi della Zanon, I monologhi e le scene, i Monologhi). Nel suo capolavoro Cronistoria Aneddotica del Teatro Nobile di Zara (1781-1881) Sabalich presenta la storia del Teatro Nobile di Zara in quarantaquattro fascicoli. Esordì nel 1879 con il monologo Le simpatie di Gemma, dedicato all'attrice Gemma Cuniberti alla quale dedicarono le loro opere Eugenio Zorzi, Paolo Ferrari, Giacinto Gallina e altri. La maggior parte delle opere teatrali di Sabalich fu rappresentata sui palcoscenici di Zara e di molte altre città italiane. Esistono inoltre notizie relative alla rappresentazione di parecchi drammi che sono andati perduti (Balić-Nižić 2008: 25-63). Una recente scoperta di opere drammatiche manoscritte, effettuata dalle autrici del presente articolo nel Fondo Sabalich della Biblioteca scientifica di Zara, offre una nuova prospettiva sull'eventuale pubblicazione di alcuni testi considerati perduti in un'edizione critica.

Nel lascito di Giuseppe Sabalich alla Biblioteca scientifica di Zara oltre a un consistente epistolario che sta a evidenziare l'interesse prolifico del nostro autore, si conservano anche le missive di Laura Zanon Paladini. Si tratta di 30 missive, di cui 26 cartoline postali e 4 lettere, scritte tra il 1898 e il 1906 (Ms. 1102/I, ZKZD). Occorre evidenziare che una delle cartoline e una lettera non sono databili. Non essendo possibile in questa sede addentrarsi nei dettagli biografici di Laura Zanon Paladini, ci si soffermerà su alcuni particolari che serviranno da ganci a cui appendere l'interpretazione del rapporto tra l'autore e l'attrice.

Laura Zanon Paladini fu una famosa attrice, nata a Venezia nel 1845, morta a Milano nel 1919. Esordì nel teatro in lingua, ma il suo vero successo cominciò grazie al passaggio al vernacolo veneziano che si dimostrò a lei più affine. Facendo parte di diverse compagnie dialettali, infine trionfò in quella di Feruccio Benini dal 1897 in poi (Varagnolo 1937). Non è da dimenticare che la Compagnia Benini accanto all'altra compagnia veneziana di Emilio Zago, era considerata la "Compagnia modello" all'epoca soprattutto per i suoi interpreti, tra i quali Feruccio Benini, Italia Benini-Sambo, Laura Zanon Paladini e Albano Mezzetti (Cauda 1914: 136-137). Morto Feruccio Benini, nel 1916 la compagnia si disperse e Laura Zanon Paladini si ritirò a Milano, dove poco dopo anch'ella morì ricordata come «attrice di mirabile festevolezza comica, di naturalezza senza eguale, di inarrivabile comunicativa, [...] una delle ultime "servette" della scena italiana.» (Annali del teatro italiano 1921: 244)

Quale fu il rapporto di Giuseppe Sabalich con quell'attrice «alta una spanna, nera come un chicco di pepe, con degli occhietti chiari, penetranti, metallici» (Levi 1907-1908: 197)? Vista la sua fortuna sui palcoscenici italiani, non sorprende che anche Giuseppe Sabalich fosse incantato dal suo modo di recitare, il che lo indusse a scrivere proprio per lei come prima opera La bela Nene. Il pubblico zaratino poté assistere alla prima di quest'opera all' Arena Vitaliani, il 23 agosto 
1897. In seguito, il monologo fu messo in scena al Teatro Goldoni di Venezia, alla Fenice di Trieste e gli anni successivi a Roma, Ancona, Milano, Genova, Bologna e Torino (Sabalich 1925: 2). Da quel momento in poi fu Laura Zanon Paladini a promuovere l'attività letteraria drammatica di Sabalich, rappresentandola in diversi teatri italiani. Un fatto ben noto, anzi rilevato da Giuseppe Sabalich stesso, è che parecchi monologhi in dialetto composti in svariati anni furono ispirati da e addirittura scritti per l'attrice, e infine, nel 1925, raccolti in un unico volume $I$ monologhi della Zanon. Si tratta di cinque monologhi: La Bela Nene, La Fiorera, I oto zorni de Gegia, La Comare e La mare de i gati (Sabalich 1925).

Benché nei contributi dedicati a Sabalich venga messa in evidenza la lunga amicizia con Laura Zanon Paladini, si è deciso di proporre una rilettura dell'epistolario esistente per recuperarne il racconto di un rapporto non sempre pacifico. Certo che, in mancanza delle missive di Sabalich, l'incompletezza dell'epistolario non ci permette d'illustrare il loro rapporto professionale fino in fondo. Tuttavia, bastano le parole di Zanon Paladini a darci un'idea di come fosse la vita teatrale dell'epoca, ma soprattutto a regalarci un cantuccio intimo nascosto dietro il palcoscenico. È certo che in questa sede il tema non sarà esaurito, perciò quanto segue costituirà una breve lettura concentrata su alcuni dei temi più ricorrenti.

Già dalla prima cartolina scritta da Pisa il 29 giugno del 1898 si capisce che l'amica Laura non fosse soltanto l'interprete dei monologhi del nostro. Infatti, lei si rivolge a Giuseppe in veste di critico sincero e onesto che non esita a rimproverargli le mancanze presenti nei suoi testi:

Lessi il monologo e francamente preferisco La Bella <sic!> Nene. Questo è troppo semplice e non c'è la trovata. Nell'altro la trovata è il tipo. Per ora lasciamola fino che a Lei o a me verrà in mente qualche cosa.

Dalle cartoline scritte in seguito è chiaro che l'attrice stesse recitando La Bela Nene in diversi teatri italiani, cosicché da Firenze il 28 gennaio del 1899 informò lo scrittore che anche lì avrebbe dato il suo monologo. Probabilmente fu Sabalich ad incitarla ripetutamente, perché nella stessa missiva Zanon Paladini cercò di spiegargli da che cosa dipendeva il programma teatrale: «Le ripeto ancora che molte volte non posso darlo perché devo favorire qualcuno della città ove mi trovo perlopiù sono giornalisti, scrittori ecc. E non si può ricusare.» Un anno dopo, scrivendogli da Trieste il 5 febbraio del 1900, cercò di nuovo di convincerlo a presentare la sua opera ovunque e qualora lei non fosse ostacolata:

L'ultima sera a Venezia feci la serata e diedi il suo monologo vi furono 4 chiamate è contento? E badi che dovei lottare con persona di Venezia che voleva ne facessi uno suo mi faceva anzi comprendere che per quell'occasione m'avrebbe fatto un piccolo presente, ma Lei sa che gli artisti sono grati alla dimostrazioni <sic!> d'affetto ma non si lasciano comprare.

Dalla missiva scritta da Trieste il 19 febbraio dello stesso anno è da presupporre che Giuseppe non smettesse di insistere per la rappresentazione del suo monologo: 
Ricevo la sua lettera e com'Ella desidera rispondo subito. Io feci il suo lavoro, prima di tutto perché mi era simpatico perché vi trovai dello spirito e perché il tipo era nuovo; in secondo luogo, Lei mi scrisse molte volte pregandomi di rappresentarlo ed io non volli mancare ad un impegno preso. Venerdì 23 si fa la mia serata e si fa il suo monologo, non è certo un regalo che mi può far cambiare opinione o mancare alla parola. Noi artisti siamo riconoscenti a chi si usa delle attenzioni ma pur sistema l'arte va sopra tutto.

Di particolar interesse è la reazione negativa dell'attrice al monologo Fiorera. Fu lei ad evidenziare uno dei principali difetti che si rivela nella monotonia del personaggio troppo simile alla Bela Nene, cosicché da Forlì il 29 novembre del 1900 espresse una forte delusione nei confronti del manoscritto ricevuto:

M'aspettavo un genere tutto diverso. Facendo ora la Fiorera direbbero che Lei si copiò da se stesso e che io faccio la copia della Nene. Assolutamente dove feci la Nene non farei questa, la critica sarebbe inesorabile per me e per Lei; dove non la feci non avrei scopo di lasciare quella nella quale oramai collo studio trovai i giusti effetti.

A giudicare da quanto letto nelle cartoline successive sembra che la discussione sulla Fiorera fosse diventata assai vivace e che Sabalich avesse insistito sull'opinione di Feruccio Benini che si evince uguale a quella di Laura Zanon:

Com'ella desiderava feci leggere la sua Fiorera al Benini ma... l'opinione che le espressi fu pure la sua: l'eguaglianza del tipo, del fatto, delle frasi porrebbero sotto la critica Lei e me. Perché dunque incorrere in ciò? (Trieste, il 18 dicembre 1900)

Una lacuna di quasi un anno, in seguito alla quale Sabalich risultò irritato, come si evince dalle missive successive. Infine, però, decise di mandare alla sua musa un altro monologo in attesa della sua opinione:

Rimasi molto meravigliata nel ricevere la sua lettera credevo proprio ch'Ella fosse in collera con me perché la sua ultima a me diretta non era troppo gentile e fu per questo ch'io non recitai più la Bela Nene temendo Ella me ne facesse un rimprovero. Ora dunque non mi resta che ringraziarla del gentile pensiero di dedicare a me la sua Fiorera ma si ricordi che non m'impegno di recitarla, come non la ricuso del tutto. Ho ricevuto il copione della Fiorera ed anche Quello dei Otto zorni de Gegia, e se vuole ch'io le parli francamente mi piace più questo che l'altro e forse se qui farò la serata tenterò questo. (Roma, 1'8 ottobre 1901)

Dal carteggio si evince l'insistere di Giuseppe sulla Fiorera anche due anni dopo, quando l'attrice nella lettera datata 15 febbraio 1902 scrisse: «Sa la mia opinione sulla Fiorera? Eppure non vuol recedere», per procedere in seguito con tono infastidito e rimproverargli di aver provato a corromperla:

Ella ha una brutta idea degli artisti! Gli crede molto venali, ciò dipende dall'aver praticato artisti di bassa sfera o miserabili a cui mancava il pane e quindi costretti a transigere su ogni mezzo pur di vivere! Se Ella si movesse da Zara e praticasse gli Artisti a Milano, Firenze, Roma, Bologna ecc: si 
persuaderebbe che denari ne spendono ma non mendicano. Con questo non le nego che quando ci viene fatto qualche omaggio specialmente in serata riesce gradito, ma ciò non ci compra! Si ricorderà d'avermi esibito un orologio d'oro perché rappresentassi la Fiorera eppure non la feci.

Tuttavia, per una migliore comprensione del rapporto tra Laura e Giuseppe è importante chiarire che nel caso di altri monologhi l'attrice cerca di rimediare i difetti indicati. Così per esempio, grazie al suo intervento, uno dei monologhi cambia il titolo dato dall'autore: «Non misi il titolo Una serva a spasso perché v'è una farsa (e l'avevamo fatto due giorni prima) intitolata Un camerier a spasso, del resto il titolo I oto zorni de Gegia è più nuovo quindi migliore.» (Da una lettera non datata). Anzi, il nostro autore rinuncia ad un suo monologo Il gatto non graffia, apprezzando il consiglio di Zanon Paladini: «Devo attaccarmi al proverbio: non lasciar la strada vecchia per la nuova!»(Genova, 11 aprile 1904). Fu proprio l'attrice, dopo la lettura del monologo Comare in cui rilevava «posizioni e frasi molto azzardate», a suggerire i cambiamenti al modello della commedia I Recini da Festa (1876) di Riccardo Selvatico (Agostini 2018):

Ella m'accenna a cambiamenti nella Comare? ...faccia. Lei la purghi un poco...ci sono delle Comari buone, oneste, vede il Benini da festa del Selvatico! E pure una comare del popolo, ma quanto cuore!.. il cuore si può trovare cercandolo anche in un assassino. (Trieste, il 15 febbraio 1902)

Dopo i cambiamenti, il monologo Comare fu recitato dalla Zanon per la prima volta al Teatro La Fenice il 4 febbraio del 1904 (Sabalich 1925: 40).

Gli esempi del carteggio citati fino ad ora evidenziano che Laura Zanon Paladini, oltre ad essere musa dello scrittore zaratino e interprete delle sue opere, partecipò attivamente al processo creativo di Sabalich e in tal modo diventò mediatrice del suo trapassare i limiti puramente locali, placando almeno in parte l'amarezza di Sabalich per la gloria mancatagli:

E rimasi a mezzo; abbandonai allora la (omesso) e mi gettai nel teatro. Scrissi per il Corazza, per il Papadopoli, per il Brunorini lavoretti che non varcarono il gran mare e rimasero tentativi di un dilettante di provincia; le mie opere furono recitate a Trieste, Padova e Venezia ma non c'era Enrico Gallina a farmi diventare celebre come fece di suo fratello Giacinto. (Perlini 1939: 35)

Senza la pretesa di un'analisi esaustiva, si evincono i rapporti concordanti ma anche contrastanti tra Giuseppe Sabalich e Laura Zanon Paladini. Certamente non estraneo alle correnti del teatro veneto dialettale, Sabalich promuove la propria produzione teatrale oltre i confini della Dalmazia ed inevitabilmente è costretto a placare la conflittualità immanente nel rapporto tra autore e attore, cercando di porre in maggior rilievo le proprie opere drammatiche. 


\section{Bibliografia}

Annali del teatro italiano. Vol. 1. (1901-1920), Milano: Aliprandi.

Agostini, Tiziana (2018). Selvatico, Riccardo <http://www.treccani.it/enciclopedia/ riccardo-selvatico_\%28Dizionario-Biografico\%29/> (30/10/2019)

Balić-Nižić, Nedjeljka (1998). Talijanski pisci u Zadru pred Proi svjetski rat 1900.1915., Rijeka: Edit.

Balić-Nižić, Nedjeljka (2008). Scrittori italiani negli anni precedenti la prima guerra mondiale (1990-1915), Roma: Società Dalmata di Storia Patria.

Cauda, Giuseppe (1914). Sulla scena e dietro le quinte, Chieri: F. Astesano.

Detoni, Narciso (1980). Giuseppe Sabalich poeta e storico zaratino in: La Rivista Dalmatica, pp. 247-265.

Levi, Cesare (1907-1908). Attrici italiani Laura Zanon - Paladini in: Rivista teatrale italiana, 12, XII, pp. 197-200.

Liburnico, N. Ledvinka (1955). G. Sabalich letterato zaratino in: La Rivista Dalmatica, pp. 67-70.

Nižić, Živko (1999). Sentimento dell'addio nella poesia in vernacolo zaratino di Giuseppe

Sabalich (1856-1928) in: Quaderni veneti, pp. 127-143.

Perlini, Marco (1939). Giuseppe Sabalich letterato e storiografo zaratino in: La Rivista Dalmatica, pp. 29-43.

Sabalich, Giuseppe (1925). I monologhi della Zanon, Venezia: Zanetti.

Varagnolo, Domenico (1937). Zanon-Paladini, Laura <http://www.treccani.it/enciclopedia/laura-zanon-paladini_\%28Enciclopedia-Italiana\%29/> (10/10/2019).

Zapperi, Ada (1966). Benini, Feruccio <http://www.treccani.it/enciclopedia/ ferruccio-benini_\%28Dizionario-Biografico\%29/> (30/10/2019)

ZKZD Ms. 1102/I 


\section{Pisma Laure Zanon Paladini Giuseppeu Sabalichu u Znanstvenoj knjižnici u Zadru}

U rukopisnoj zbirci Znanstvene knjižnice u Zadru sačuvana je ostavština Giuseppea Sabalicha, talijanskog kulturnog povjesničara i književnika rođenoga 13. veljače 1856., umrloga 13. rujna 1928. godine u Zadru. Dio ostavštine su i mnogobrojna pisma velikog broja pošiljatelja koja svjedoče o raznolikoj i plodnoj djelatnosti ovog Zadranina na prijelazu iz 19. u 20. stoljeće. Među velikim brojem pošiljatelja u Sabalichevu epistolariju nalazi se ime Laure Zanon Paladini, poznate i uspješne onodobne talijanske glumice, članice venecijanskog kazališta Feruccia Beninija. Riječ je o pet pisama te dvadeset i šest dopisnica datiranih između 29. lipnja 1898. i 14. kolovoza 1906. godine. Analiza pisama na relaciji Zanon Paladini - Sabalich i njihovo tumačenje kroz prizmu dosadašnjih saznanja o Sabalichevoj kazališnoj aktivnosti i bliskom profesionalnom odnosu s ovom talijanskom glumicom otkriva kako je Laura, osim uloge muze i izvoditeljice Giuseppeovih tekstova, imala istaknutu ulogu u samom procesu književnog stvaranja. Svojim kritičkim savjetima utječe na preinake Sabalichevih tekstova, koristi glumačko iskustvo kako bi ih prilagodila scenskom izvođenju čime doprinosi talijanskoj fortuni Giuseppea Sabalicha.

Ključne riječi: Giuseppe Sabalich, Laura Zanon Paladini, epistolarij, dijalektalni teatar 
